\title{
Editorial on future of advanced robotics and its applications
}

\section{Introduction}

The future of Robotics is not only fascinating but also intriguing. Below are few of many applications of advanced robots that will aid us to achieve; that may be either very tough or almost impossible tasks for humans.

\section{Ocean robots could help humans with dangerous job}

Exploring the ocean and repairing underwater structures can be dangerous work. Exploring deep into oceans is not only perilous but also may cause high risk on health. Researchers are designing robots that could do these jobs. One of the examples that were developed was a kind of robot that looks like a snake. It would repair and inspect oil rigs. Its thin and flexible shape would allow it to go where divers cannot. Tiny submarines known as Eco subs could help scientists study and explore the ocean. One person could control a group of eco subs. Each sub would have different sensors. The sensors would work together to collect information. Unmanned boats could help with data collecting and study for further use.

\section{Robotic house flies that could help find survivors in disasters}

House Flies are known for their ability to squeeze into small spaces and with winged and miniature size that would reach to most of the corner. That's why scientists are using technology inspired by House Flies exoskeletons to develop robots. These robots could be used to search disaster areas. The robotic House Flies would be able to squeeze through small spaces in the rubble and find possible survivors. Scientists have been studying live flies to see just how they manage to slip between small spaces. Their findings allowed them to create piece to make a robotic flies that could save lives.

\section{Robots that can help to discover inside volcanoes that humans cannot}

National Aeronautical and Apace Administration (NASA) known mostly for sending objects into space, has developed a robot that can explore deep inside active volcanoes. The two robots, named Volcano Bots, can climb along walls and explore the fissures within a volcano. The hope is that the Volcano Bots will be able to help scientists better understand how volcanoes erupt.

\section{Robot that could perform household day to day task}

Would you like a helper robot at home? A robot that follows you, lights up any room you're in, or moves your flowers to follow the sunlight? This prototype robot is designed like a docking station. Owners could purchase attachments to connect it, allowing it to perform all sorts of tasks.

\author{
Volume 3 Issue 2 - 2017 \\ Manu Mitra \\ Department of Alumnus with Electrical Engineering, University \\ of Bridgeport, USA
}

Correspondence: Manu Mitra, Department of Alumnus with Electrical Engineering, University of Bridgeport, USA, Emailmmitra@my.bridgeport.edu

Received: September 20, 2017 | Published: October 02, 2017

\section{Robots that become adaptive furniture}

Researchers in Switzerland have created a robot that may change the landscape of our living rooms. Room bots are morphing robots, able to take the shape of objects such as chairs, tables, and pews. The robots are made of interlocking modules that shift and rotate, allowing the machine to easily change shape and move into the desired position and configuration. The researchers plan to use Room bots to create furniture that assists elderly people by adapting to their needs. For example, the robot might reconfigure to provide a place to sit, or lend steady support to someone who has fallen down. Therefore, dynamically growing technology of robotic industry is going to be investable and definitely will re shape the near future of human kind not only just in technological perceptive but also in medical, business and our day to day life. Our International Robotics and Automation Journal (IRATJ) ISSN: 2574-8092 with its international caliper and scope, we cover a wide range of research lines from design, construction, and operation and beyond industrial, domestic/household. Finally, this journal will be committed to continue its mission to focus as with the international research community to achieve the clearest possible scientific picture on the coming up horizon of Robotics for a better life of human kind in particular. The journal cordially invites Research Articles, Review Articles, Short Communications, Case Reports, Mini- Reviews, opinions, Letter to Editors Etc. for the latest ongoing and future research. The journal will continue to acts to serve research community and public.'

\section{Acknowledgments}

None.

\section{Conflict of interest}

Author declares that there is none of the conflicts.

\section{References}

1. The 12 Biggest Breakthroughs in Robot Technology. 2017. 